\title{
Effect of thermocouple size on the measurement of exhaust gas temperature in internal combustion engines
}

\author{
Nick Papaioannou, Felix Leach, Martin Davy \\ University of Oxford
}

\begin{abstract}
Accurate measurement of exhaust gas temperature in internal combustion engines is essential for a wide variety of monitoring and design purposes. Typically these measurements are made with thermocouples, which may vary in size from $0.05 \mathrm{~mm}$ (for fast response applications) to a few millimetres. In this work, the exhaust of a single cylinder diesel engine has been instrumented both with a fast-response probe (comprising of a $50.8 \mu \mathrm{m}, 127 \mu \mathrm{m}$ and a $254 \mu \mathrm{m}$ thermocouple) and a standard $3 \mathrm{~mm}$ sheathed thermocouple in order to assess the performance of these sensors at two speed/load conditions. The experimental results show that the measured timeaverage exhaust temperature is dependent on the sensor size, with the smaller thermocouples indicating a lower average temperature for both speed/load conditions. Subject to operating conditions, measurement discrepancies of up to $\sim 80 \mathrm{~K}$ have been observed between the different thermocouples used. Thermocouple modelling supports the experimental trends and shows that the effect of conduction is inversely proportional to the thermocouple junction size-an effect attributed to changes in the thermal inertia of the device. This conduction error is not typically considered in the literature for exhaust gas temperature measurement. Modelling results also show that radiative heat transfer is small compared to the effect of conduction on the measurements. Finally, a new dynamic response thermocouple compensation method is presented, in order to correct for the dynamic error induced by the thermocouples. This technique recovers the "true" gas temperature with a maximum error of $\sim 1.5-2 \%$ in peak temperature depending on speed/load conditions.
\end{abstract}

\section{Introduction}

Accurate measurement of exhaust gas temperature (EGT) in internal combustion engines is important for a wide range of monitoring and design purposes. Unreliable EGT measurements can lead to component damage from excessive thermal loading [1], or to the underutilisation or inefficient use of turbocharger and after-treatment systems [2-4]. The conversion efficiency of diesel oxidation catalysts and selective catalytic reduction (SCR) systems is dependent on their operating temperature and consequently to the exhaust gas temperature. The regeneration of diesel particulate filters (DPFs) is achieved by retarding the post-injection timing thus increasing the temperature of the exhaust gases [5]. Accurate monitoring of the EGT, and thus being able to optimise the regeneration event, has the potential to improve fuel efficiency.

Accurate knowledge of exhaust gas temperature is also important for heat transfer modelling of exhaust systems and CFD model

Page 1 of 12 development and validation [6]. In addition, energy balance studies on internal combustion engines highlight the need for accurate EGT, especially when investigating the effects of different combustion strategies [7-11].

The measurement of EGT in internal combustion engines usually involves the use of thermocouples due to their low cost, simplicity and robustness at elevated temperatures. In order to capture the transient behaviour of the EGT in specialised research applications, fine-wire thermocouples, which can vary in size from 0.001 " to 0.032 ", are commonly employed $[11,12,13,14]$. However, larger, more robust thermocouples (e.g. $3 \mathrm{~mm}$ sheathed probes) are typically used for conventional test-bed applications, to the detriment of the measurement system's dynamic response.

The dynamic response of a thermocouple to temperature fluctuations is governed by its time-constant $(\tau)$ defined as:

$$
\tau=\frac{m c_{p}}{h A_{s}}
$$

Where $m$ and $c_{p}$ are the mass and the heat capacity and $h$ and $A_{s}$ the heat transfer coefficient and the surface area of the thermocouple junction. Dupont [15], Heitor [16], Hopkins [17] and their respective co-workers describe a number of different experimental techniques that may be used to identify a thermocouple's time-constant in steady flow. However, as can be seen by the presence of the convective heat transfer coefficient in Eqn (1), the thermocouple time-constant is flow-dependent and the results of such techniques are not applicable for a wide range of flow conditions. In order to correct for this dynamic error in situ, several methods have been developed that use the output of two different size thermocouples to estimate the instantaneous thermocouple time-constants and thus correct the dynamic error [13, 14, 18-20].

In addition to the potential errors associated with an uncertain dynamic response, thermocouple temperature measurements are commonly subject to additional potential sources of error; conduction and radiation, which are dependent on the geometrical properties of the sensor, the characteristics of the flow to be measured and the installation of the sensor relative to the flow (as well as potential errors relating to the catalysis of the thermocouple metals which are typically very small and hence will be disregarded in this study).

Measurement errors introduced due to radiative heat transfer [21-23], and slow dynamic response [13,14, 18-20] of thermocouple sensors in temperature measurement have been widely covered in the 
literature. On the other hand, thermocouple conduction error is less commonly studied and the published work is mostly limited to temperature measurements in steady flows [21, 22, 24, 25]. Conduction error is a consequence of the temperature gradient along the axis of the sensor, resulting in a heat flow away from the measurement location, and thus to a discrepancy between the fluid and the sensor's apparent temperature. It has been shown that this error can be reduced by either, increasing the sensor's immersion length, or by reducing its diameter (and hence its conduction area) $[22,25]$. However, these conduction error reduction techniques are not always applicable, depending on the application, and therefore an understanding of the conduction error is required in order to achieve an accurate measurement.

This work combines experimental and numerical studies to assess the performance of different size thermocouples on the measurement of EGT in internal combustion engines and to quantify the relative importance of the different sources of error, namely; conduction, radiation and dynamic response.

\section{Experimental apparatus and methodology}

\section{Engine and Instrumentation}

A single cylinder diesel engine was used to provide a pulsating flow of unsteady temperature [26]. Two types of thermocouples have been used; fast response and mineral sheathed thermocouples. Three barewire K-type thermocouples, of varying junction diameters $(50.8 \mu \mathrm{m}$ (T1), $127 \mu \mathrm{m}$ (T2) and $254 \mu \mathrm{m}$ (T10)), were combined in a single thermocouple probe (hereafter called 3-TC probe) as shown in Figure 1. A $3 \mathrm{~mm}$ mineral sheathed $\mathrm{K}$-type thermocouple, representing conventional engine instrumentation, was used as a reference EGT sensor. The manifold wall temperature, required for the estimation of radiation and conduction losses, was measured by an embedded $\mathrm{K}$ type thermocouple. The relative location of each of the sensors can be seen in Figure 2.

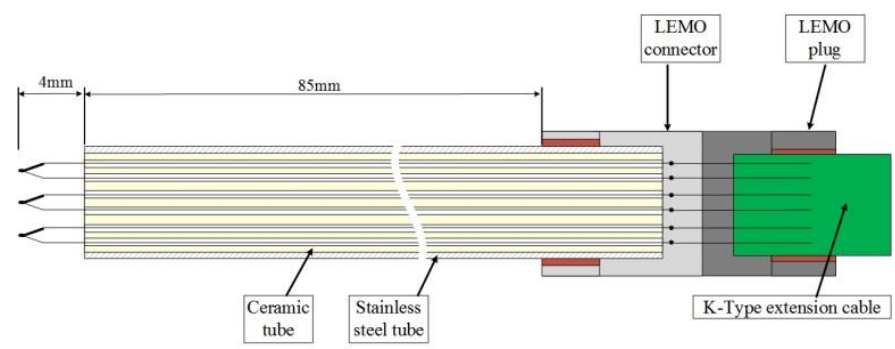

Figure 1: Three thermocouple probe schematic (not to scale)

The 3-TC probe was manufactured following the design of Kar et al., [13]. The thermocouple pairs were passed through a six-hole ceramic tube to hold them in place. In order to avoid damage, the ceramic tube was inserted in a $3 \mathrm{~mm}$ stainless steel tube and bonded using a high temperature cement paste. The thermocouples were then soldered onto the pins of a LEMO connector which was fixed at the end of the probe. K-type extension wire was used for the signal transmission to the amplifier eliminating the need for cold junction compensation at the LEMO plug (it is assumed that the plug is isothermal and follows the law of intermediate metals [27]).

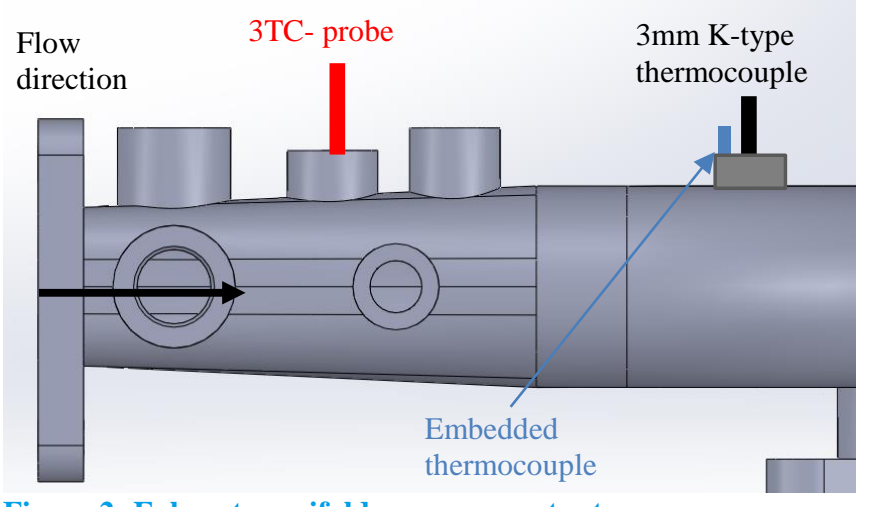

Figure 2: Exhaust manifold measurement set-up

All thermocouple signals were amplified $(\times 200)$ before logging using an INA 110 instrumentation amplifier. The cold junction compensation was measured with an external thermocouple at the input of the amplifier (Figure 3). The thermocouple reader and the cold junction thermocouple have been calibrated in a water bath and the error associated with the cold junction measurement is $\pm 0.2 \mathrm{~K}$ at $95 \%$ confidence limits. After amplification the thermocouple signals were logged using an AVL Indicom high-speed data acquisition (DAQ) system at a resolution of $1 \mathrm{CAD}$. The conversion from thermocouple voltage to temperature was done offline with the use of K-type polynomials based on the ITS-90 temperature scale and is accurate to within -0.05 to $0.04 \mathrm{~K}$ [28]. The thermocouple measurement set-up can be seen in Figure 3.

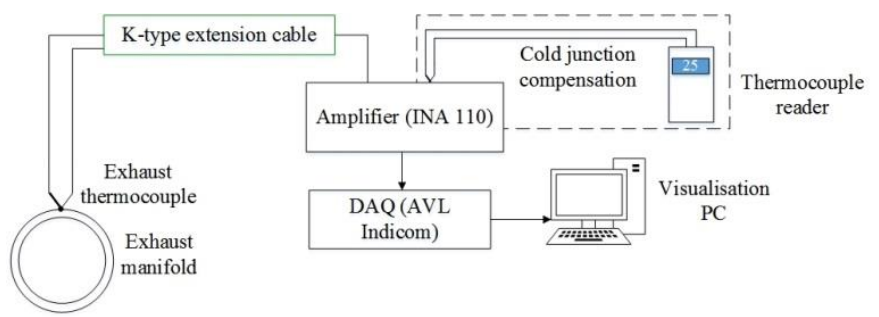

Figure 3: Thermocouple measurement and data acquisition configuration.

\section{Calibration}

All thermocouples were initially checked for linearity using a water bath calibration in the range of 298-362 K, with good agreement. As exhaust temperatures can range from $573-773 \mathrm{~K}$ for the conditions presented here, a miniature oven capable of simultaneously supporting the 3-TC probe and the reference thermocouple was designed and built for higher temperature calibration. The oven was designed so as to minimise the measurement volume once the two sensors were installed, thereby minimising potential errors arising from temperature gradients due to natural convection [29]. Experiments were performed in the range of $473-773 \mathrm{~K}$ and the measurement process repeated three times. The 3-TC probe signals were first amplified (using the same process presented above) and then read into an oscilloscope. The reference thermocouple signal was logged using a standard thermocouple reader. The thermocouple signals were logged once the reference thermocouple reached the required temperature and all readings were stable. 


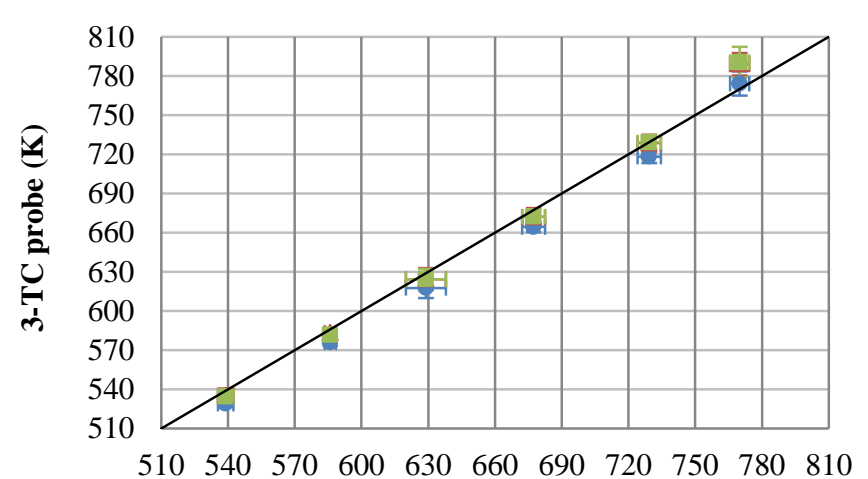

Tref $(\mathbf{K})$

- $\mathrm{T} 2 \Delta \mathrm{T} 5 \quad \mathrm{~T} 10$

Figure 4: Miniature oven calibration results. Error bars indicate the $95 \%$ confidence limits.

The results of the calibration are presented in Figure 4 where the yaxis indicates the readings for the 3-TC probe thermocouples and the $\mathrm{x}$-axis the reference thermocouple readings. The error bars represent the $95 \%$ confidence limits and the black line the unity slope line of the reference thermocouple. The observed discrepancies at the highest temperatures are attributed to the much faster cooling rate of the oven compared to the rate of heating input at these temperatures. Consequently, due to the higher thermal mass of the reference thermocouple this resulted in a lower apparent temperature compared to the fine-wire thermocouples.

\section{Engine test conditions}

The engine was operated at two different speed/load conditions in order to assess the response of the different thermocouple sensors. The engine operating conditions can be seen in Table 1. Once the engine reached stable operation, judged as being when the reference $3 \mathrm{~mm}$ thermocouple reading had achieved a steady state, the exhaust temperature signals were logged for 300 consecutive cycles. An ensemble average for each thermocouple signal was then calculated using AVL Concerto software and converted to temperature using the process described in the previous section.

Table 1: Engine test operating conditions

\begin{tabular}{|c|c|c|}
\hline Test point & $\begin{array}{l}1500 \mathrm{rpm} / 6.8 \\
\text { bar nIMEP }\end{array}$ & $\begin{array}{l}1750 \mathrm{rpm} / 13.5 \\
\text { bar nIMEP }\end{array}$ \\
\hline Engine speed (rpm) & 1500 & 1750 \\
\hline nIMEP (bar) & 6.8 & 13.5 \\
\hline $\begin{array}{l}\text { Approximate exhaust } \\
\text { temperature }(\mathrm{K})\end{array}$ & 623 & 753 \\
\hline Wall Temperature (K) & 506 & 589 \\
\hline
\end{tabular}

Page 3 of 12

\section{Numerical methodology}

\section{Lumped capacitance thermocouple model}

The thermocouple response to fluctuations in exhaust gas temperature was modelled using a 1-D lumped capacitance approach. Figure 5 shows an energy balance applied to a control volume around a thermocouple junction in a cross-section of exhaust pipe. It is assumed that there is a uniform temperature distribution over the cross section of the exhaust and that there is no variation in temperature along the radial and circumferential direction of the thermocouple. It is also assumed that the exhaust wall temperature is constant (the engine is operating at steady-state).

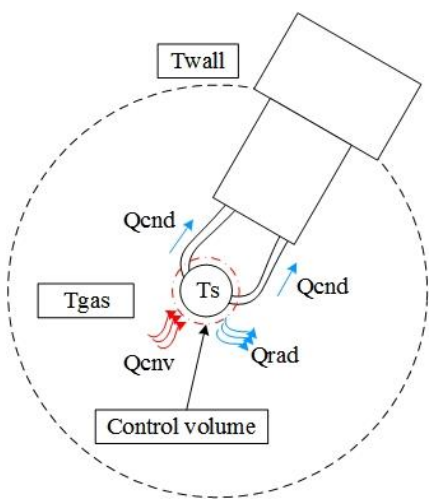

Figure 5: Energy balance for a typical thermocouple junction in internal heat flow measurement. The red dashed line indicates the boundaries of the control volume.

When the thermocouple is exposed to the hot gas flow, heat will be convected from the hot gas to the thermocouple junction. However, as the surrounding wall temperature is at a lower temperature than the gas (refer to Table 1), heat also will be transferred towards the colder surroundings both via conduction and radiation. Mathematically, the response of the thermocouple junction to a temperature fluctuation can thus be expressed following the equation below:

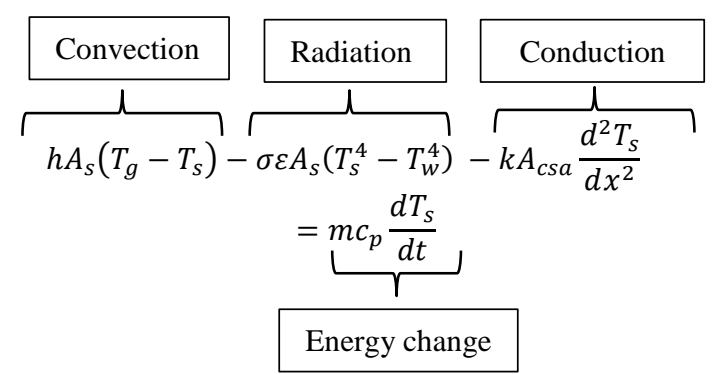

where $h$ is the convective heat transfer coefficient, $\sigma$ is the StefanBoltzmann constant, $\varepsilon$ the emissivity of the thermocouple material and $k, m$ and $c_{p}$ are the thermal conductivity, mass and heat capacity of the thermocouple material respectively. $A_{s}$ is the junction surface area and $A_{c s a}$ denotes the cross-sectional area of the thermocouple wire. The gas temperature is given by $T_{g}$, the temperature measured by the thermocouple is given by $T_{s}$ and the exhaust manifold wall temperature is given by $T_{w}$. This equation is solved at each time-step to give the temperature of the thermocouple $T_{S}$.

The size and geometry of each thermocouple's measurement junction were determined by photomicrography. The results are summarised 
in Table 2, which also details the relevant Nusselt number correlation used for each thermocouple and the calculated Biot number (Bi $\ll<0.1$ thus confirming the validity of the lumped capacitance approach). Note that the precise size of the sensing junction of the butt-welded thermocouples is difficult to establish by optical techniques. Accordingly, the junction size of the butt-welded thermocouples reported in Table 2 has been corrected to match experimental data.

Table 2: Characteristics of modelled thermocouples

\begin{tabular}{llll}
\hline $\begin{array}{l}\text { Thermocouple } \\
\text { diameter }\end{array}$ & $\begin{array}{l}\text { Nusselt } \\
\text { correlation }\end{array}$ & $\begin{array}{l}\text { Junction } \\
\text { geometry }\end{array}$ & $\begin{array}{l}\text { Biot } \\
\text { number }\end{array}$ \\
\hline $0.0508 \mathrm{~mm}(\mathrm{~T} 2)$ & Sphere & Bead & 0.000165 \\
$0.127 \mathrm{~mm}(\mathrm{~T} 5)$ & Tube & Butt-welded & 0.000325 \\
$0.254 \mathrm{~mm}(\mathrm{~T} 10)$ & Tube & Butt-welded & 0.000396 \\
$3 \mathrm{~mm}$ (Tref) & Tube & Sheath & 0.000528 \\
& & & \\
\hline
\end{tabular}

Air was used as the working fluid in all simulations. The thermophysical properties $\left(k, c_{P}\right.$ and $\left.\mu\right)$ of air were calculated at each timestep based on published data and the corresponding exhaust gas temperature predicted by a one-dimensional engine model which is discussed in the following subsection of this work and which also provided the flow velocity necessary for the calculation of the Reynolds and Nusselt numbers. Nusselt number is calculated, for all geometries, from the correlation of Whitaker [30] who suggested, that Equation 3 could be used for both spherical geometries and tubes in a crossflow.

$$
N u=2+\left(0.4 \operatorname{Re}^{\frac{1}{2}}+0.06 \operatorname{Re}^{\frac{2}{3}}\right) \operatorname{Pr}^{0.4}\left(\frac{\mu_{b}}{\mu_{0}}\right)^{\frac{1}{4}}
$$

where $R e$ is the Reynolds number, $\operatorname{Pr}$ is the Prandtl number, and $\frac{\mu_{b}}{\mu_{0}}$ is the ratio of the dynamic viscosity at the surface of the body to the dynamic viscosity at free stream conditions. Note that the viscosity ratio in Equation (3) has been calculated at every time-step to account for the change in thermocouple surface and gas bulk temperatures and has been shown to remain within the specified limits defined by Whitaker (e.g. 0.25-5.2 for a cylinder and 1.0-3.2 for a sphere).

K-type thermocouples are comprised of nickel based alloys - the positive leg is made of Chromel and the negative leg of Alumel [12] - accordingly, the material properties used for the fine-wire thermocouple simulations, were those of nickel. Table 3 details the thermo-physical properties of nickel, which can be assumed to be independent of temperature (which has been done in the present work). With regards to the emissivity, experimental work by $\mathrm{Fu} e t$ al., [31] reported an increase in the emissivity of a soot-deposited nickel substrate $(\sim 0.895)$ relative to the emissivity of a deposit-free, oxidised nickel substrate of $(\sim 0.58)$ at $743 \mathrm{~K}$. Therefore, the emissivity values presented by Fu et al., [31] (for a soot-deposited substrate) have been used for all thermocouples.

Due to the composite structure of the reference thermocouple, a finite difference 1-D model, as described in [32], was used initially to access the effect of the sheathed construction on the response of the Page 4 of 12 sensor. However, comparison against the lumped capacitance model applied to the sheathed geometry (assuming a uniform nickel construction) resulted in insignificant differences between the two methods, thus the reference thermocouple was also modelled following the lumped capacitance approach.

Table 3: Thermo-physical properties of nickel

\begin{tabular}{lcc}
\hline Property & Value & Units \\
\hline Density $(\rho)$ & 8908 & $\mathrm{~kg} / \mathrm{m}^{3}$ \\
Heat capacity $\left(c_{p}\right)$ & 440 & $\mathrm{~J} / \mathrm{kgK}$ \\
Thermal conductivity $(k)$ & 90 & $\mathrm{~W} / \mathrm{mK}$ \\
Emissivity $(\varepsilon)$ & $0.895^{*}$ & - \\
\hline Fu et al., $[31]$ & &
\end{tabular}

\section{One-dimensional engine modelling}

A commercially available one-dimensional code [33] was used to calculate the instantaneous exhaust gas velocity and temperature $\left(T_{g}\right)$ at the experimental test conditions. The necessary boundary conditions (i.e. crank angle resolved intake and exhaust pressures) were set using experimental measurements from fast response pressure transducers. In order to reduce the modelling complexity, the modelled length of the exhaust manifold was limited to the location of the reference thermocouple sensor (Figure 2).

Validation of the 1-D model was carried out against experimental values of maximum cylinder pressure, intake flow rate, peak cylinder pressure and pumping mean effective pressure (pmep) for the conditions shown in Table 1. The results of the validation, for the aforementioned parameters can be seen in Figure 6. In general there is a very good agreement with the experiment, with some deviation arising for the pmep values ( $\sim 0.05$ bar and $\sim 0.1$ bar for the higher and lower load cases respectively). These deviations are a result of the intake pressure conditions imposed on the model in order to match the experimental engine flow rate for the conditions shown in Table 1 , which, are not expected to have a significant effect on the simulated exhaust gas temperature but could affect the exhaust gas velocity profile. However, for the purpose of this work these discrepancies were considered acceptable. Due to the nature of this study, the validation of the modelled exhaust gas temperature was not possible as the uncertainties associated with the thermocouple sensors are not known.

\section{Results and Discussion}

\section{Experimental results}

\section{Engine testing}

In this section the experimental data from the 3-TC probe and the 3 $\mathrm{mm}$ sheathed reference thermocouple are presented and compared at the two different speed/load points. Figure 7 shows the measured exhaust gas temperature at the $1500 \mathrm{rpm} / 6.8$ bar nIMEP condition. The two dashed lines indicate the time of exhaust valve opening (EVO) and exhaust valve closing (EVC) events. As expected, the T2 
thermocouple - having the smallest time constant - is able to capture the blowdown event more clearly thus resulting in a higher temperature reading compared to the other two fine-wire thermocouples. It is also interesting to note that due to its lower time constant, the T2 thermocouple cools more rapidly, during the period that the exhaust valves are closed, thus resulting in the lowest apparent temperature at the end of the cycle. These effects are lessened as the size of the junction is increased.
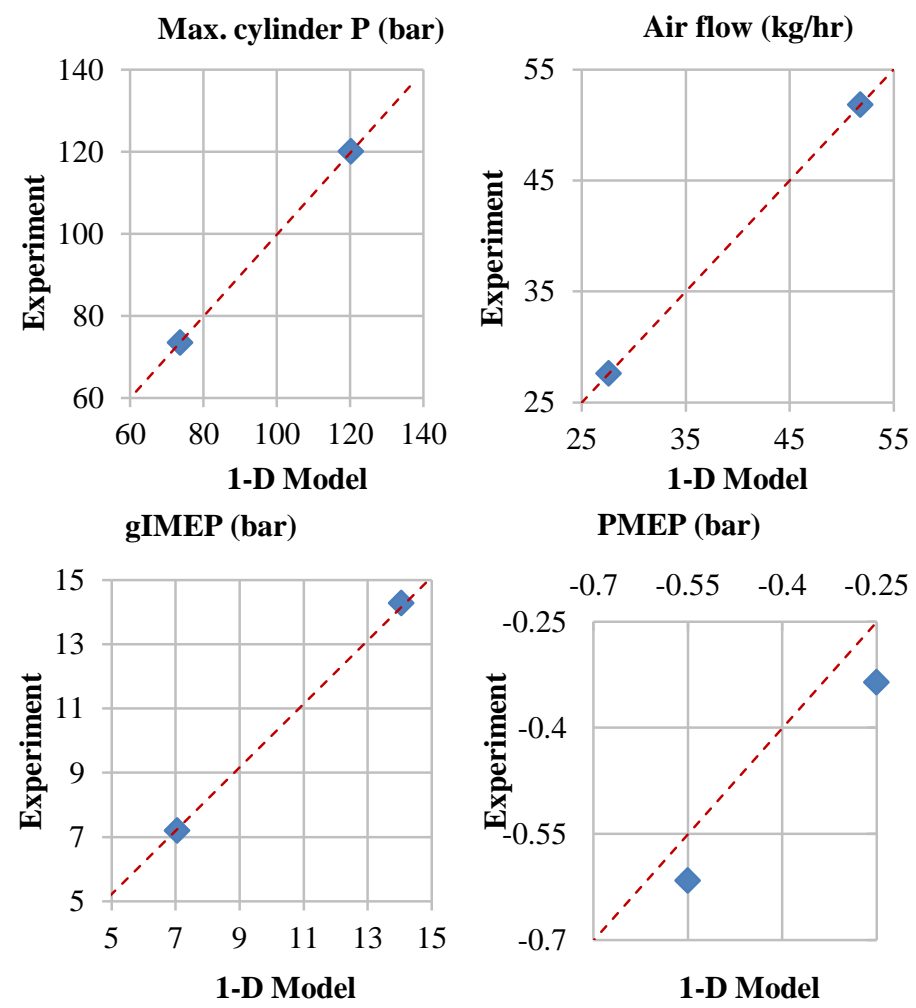

Figure 6: Validation results for the 1-D engine model. The $\mathrm{x}$-axis corresponds to the 1-D model results and the $y$-axis to the experimental measurements. The dotted lines indicate the unity slope line.

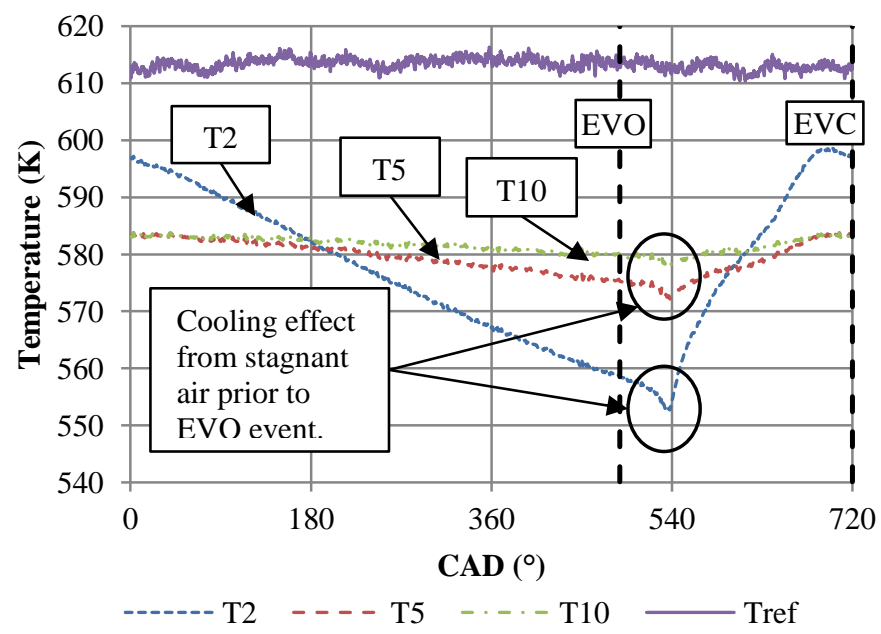

Figure 7: Experimental exhaust temperature measurements for the 3-TC probe and the reference thermocouple at $1500 \mathrm{rpm} / 6.8$ bar nIMEP point.

Page 5 of 12
Worthy of note is the sudden cooling period prior to the rapid increase in temperature that is captured by all thermocouples of the 3TC probe at approximately $540{ }^{\circ} \mathrm{CA}$. Kar et al. [13] also observed this cooling trend suggesting that it is caused by a combination of two phenomena: a) prior to EVO the column of air between the sensor and the valves is exchanging heat with the surroundings and when the EVO event occurs this "cooler" gas precedes the hot gases exiting the cylinder thus resulting in the measured cooling spike, and b) while the valves are closed they will exchange heat with the cylinder head and during the EVO period, where the flow is choked and the heat transfer very high, the first hot gases leaving the cylinder will exchange heat with the colder valves, resulting in a lower temperature. These trends discussed are the same at the 1750 $\mathrm{rpm} / 13.5$ bar nIMEP conditions and so are omitted for brevity.

The results present in Figure 7 show an unexpected behaviour in terms of the response of the different thermocouple sensors. The reference thermocouple indicates an apparent temperature of $613 \mathrm{~K}$, a reading that is significantly higher than both the instantaneous and the time-averaged temperatures measured with the 3-TC probe. Since conduction and radiation errors in temperature measurement are dependent on sensor size (the immersion length for both probes is the same) the opposite trend might reasonably be expected. Repeated experiments with a second 3-TC probe confirmed the result. The position of the two probes was also swapped to ensure that these observations were not a result of the probe location. Figure 8 presents the measured time-average temperatures for the 3-TC probe and the reference thermocouples for the two speed/load conditions. Note that at the higher speed and load condition the magnitude of the discrepancy between the fine-wire and sheathed thermocouples is of the order of $80 \mathrm{~K}$.

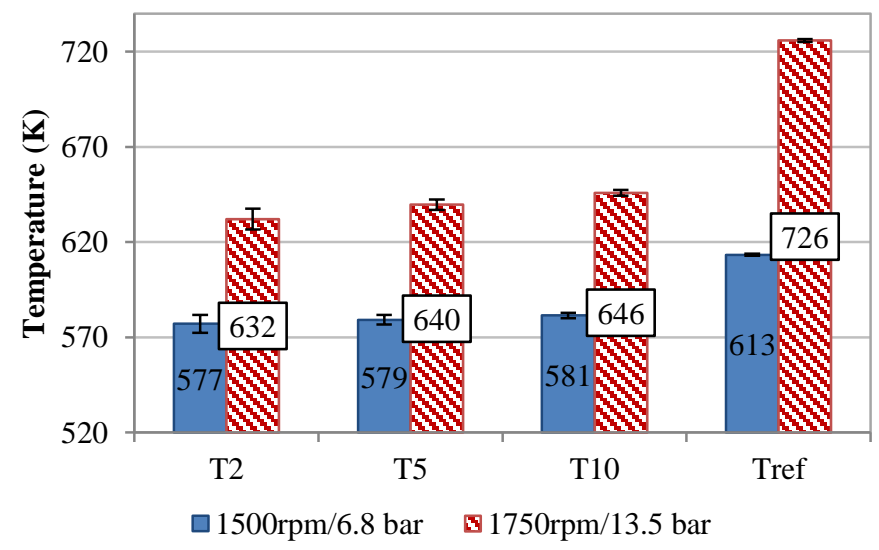

Figure 8: Experimental time-average exhaust temperatures for the different thermocouple sizes for both speed/load conditions. The blue colour indicates the $1500 \mathrm{rpm} / 6.8 \mathrm{bar}$ nIMEP and the red colour the $1750 \mathrm{rpm} / \mathbf{1 3 . 5}$ bar nIMEP conditions. The error bars indicate the $95 \%$ confidence limits.

\section{Thermocouple modelling results}

In this section, the authors present the results of the lumped capacitance modelling for the three different size thermocouples of the 3-TC probe and also for the reference thermocouple. The simulations were carried out in three stages in order to better understand the observed experimental data: firstly, the thermocouple transient response to a time varying input gas temperature $\left(T_{g}\right)$ and flow velocity from the 1-D engine simulation was predicted in the 
absence of radiation and conduction. Secondly, the simulation was expanded to include the radiation term, and finally the model was expanded to include radiation and conduction. In each case, the model was run until the temperature at the start of the cycle converged to the temperature at the end of the cycle. All data presented are converged values.

\section{No radiation or conduction terms}

Ignoring the radiation and conduction terms, Equation (2) is discretised as:

$$
T_{s}(t+1)=T_{g}(t)+\left[T_{s}(t)-T_{g}(t)\right] e^{-\frac{\Delta t}{\tau}}
$$

where the time constant $(\tau)$ is given by Equation (1) and $\Delta t$ is the size of the time-step [32].

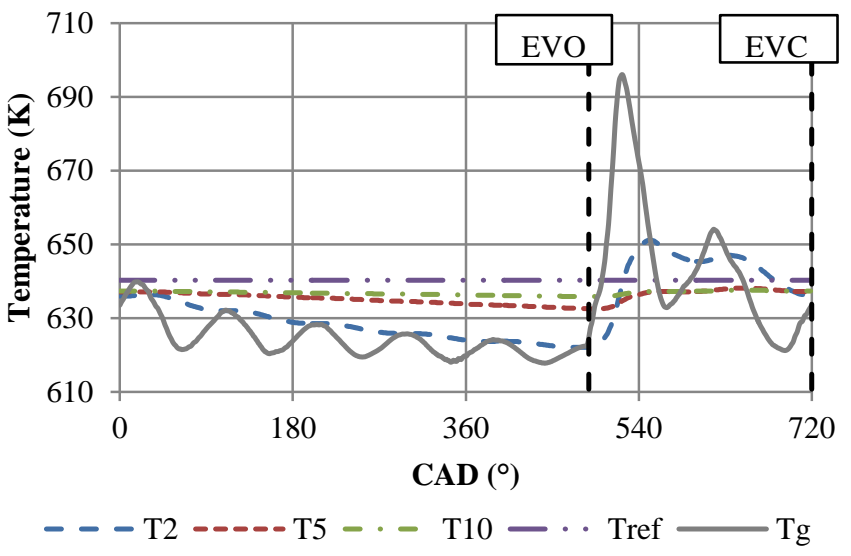

Figure 9: Simulated thermocouple results for the $1500 \mathrm{rpm} / 6.8$ bar nIMEP case (Equation 4). Effects of radiation and conduction are not included.

The model results of Equation (4) for the different thermocouples at the $1500 \mathrm{rpm} / 6.8$ bar nIMEP are shown in Figure 9 where the solid line indicates the modelled gas temperature from the 1-D engine model. The observed undulations are a result of the pressure pulses travelling along the length of the manifold - these undulations are captured by the fast response pressure transducers that are used as an input into the 1-D model-a detailed discussion of the exhaust pressure fluctuations from this engine is included in [34]. The results clearly indicate that as the size of the thermocouple increases (and consequently the time-constant) the temperature signal is significantly attenuated, as expected. As an example, the model results for the $\mathrm{T} 2$ thermocouple indicate an average time constant of $\bar{\tau}=19 \mathrm{~ms}$ over the cycle duration, which corresponds to a frequency response of $\sim 55 \mathrm{~Hz}$. Consequently, the T2 thermocouple is not able to capture the temperature fluctuations associated with the pressure pulsations occurring at $\sim 100 \mathrm{~Hz}$. Similarly the maximum temperature is also significantly under predicted, with the T2 thermocouple indicating a peak temperature of $\sim 645 \mathrm{~K}$ compared to the "true" peak temperature value of $\sim 695 \mathrm{~K}$. These observations highlight the dynamic error that is induced by thermocouple measurements of unsteady temperature flows; this dynamic error is expected to be pronounced in exhaust gas temperature measurements as the fragility of fine-wire thermocouples and the harshness of the environment, forces a compromise between frequency response and robust measurements - dictating a minimum junction diameter of 0.254 $0.508 \mathrm{~mm}[19]$.

In agreement with the experimental results presented in Figure 8, the 3-TC probe thermocouples are predicted to result in lower timeaverage temperatures compared to the reference thermocouple. However although this trend is correct, the absolute differences between the modelled and experimental values are quite different. More specifically, the results of Equation (4), for all thermocouples, result in an overestimate of the time-average temperature $\left(\bar{T}_{s}\right)$ compared to the time-averaged gas temperature $\left(\bar{T}_{g}\right)$ that is input. These differences can be seen in Table 4 for both speed/load conditions modelled.

Table 4: Modelled time-average temperature results: No radiation or conduction terms (Equation 4) for the two speed/load conditions

\begin{tabular}{cccccc}
\hline Conditions & $\overline{\boldsymbol{T}}_{\boldsymbol{g}}$ & $\overline{\boldsymbol{T}}_{\mathbf{2}}$ & $\overline{\boldsymbol{T}}_{\mathbf{5}}$ & $\overline{\boldsymbol{T}}_{\mathbf{1 0}}$ & $\overline{\boldsymbol{T}}_{\boldsymbol{r e f}}$ \\
& & & & & \\
\hline $1500 \mathrm{rpm} / 6.8$ bar IMEP & $630 \mathrm{~K}$ & $632 \mathrm{~K}$ & $635 \mathrm{~K}$ & $637 \mathrm{~K}$ & $639 \mathrm{~K}$ \\
& & & & & \\
$1750 \mathrm{rpm} / 13.5$ bar IMEP & $706 \mathrm{~K}$ & $712 \mathrm{~K}$ & $716 \mathrm{~K}$ & $718 \mathrm{~K}$ & $722 \mathrm{~K}$ \\
& & & & & \\
\hline
\end{tabular}

The discrepancies observed between the input and predicted timeaveraged temperatures shown in Table 4 are an artefact of the thermocouples' thermal inertia. By definition, as the thermal inertia of a body increases the slower it can respond to temperature changes and thus the longer it takes to reach equilibrium. Similarly, upon equilibrium, a larger thermal inertia implies a higher amount of thermal energy stored within the body and consequently a higher apparent body temperature. Therefore, as the T2 thermocouple has the smallest inertia is able to follow the temperature fluctuations during the cycle much closer and reach a steady state condition much quicker. However, since its thermal mass is not zero, once equilibrium is reached (i.e. the temperature at the beginning and the end of the cycle is the same), its apparent temperature $\left(\bar{T}_{2}\right)$ will be higher than the gas temperature $\left(\bar{T}_{g}\right)$ with the difference indicating the extra energy required to heat its thermal mass.

\section{Radiation terms included}

Equation (5) shows the discretised solution of Equation (2) for $T_{S}$ with the radiation term included and the conduction term ignored.

$$
\begin{aligned}
T_{S}(t+1)=T_{S}(t)+ & \frac{\Delta t}{m c_{p}}\left[h A_{S}\left(T_{g}(t)-T_{S}(t)\right)\right. \\
& \left.-\sigma \varepsilon A_{S}\left(T_{s}^{4}(t)-T_{w}^{4}\right)\right]
\end{aligned}
$$

From Equation (5) it is clear that the radiative term will be dependent on the exposed surface area of the body (assuming a constant wall temperature). The flow conditions will not have an effect on the energy transferred by radiation. Figure 10 shows the effect of radiation (Equation 5) in terms of time-average values for the modelled thermocouples for the $1500 \mathrm{rpm} / 6.8$ bar nIMEP case. The wall temperature was fixed at $T_{w}=509 \mathrm{~K}$. Under the $1750 \mathrm{rpm} / 13.5$ bar nIMEP case the radiation errors for each thermocouple were of the same order of magnitude as those presented in Figure 10 and have thus been omitted. 


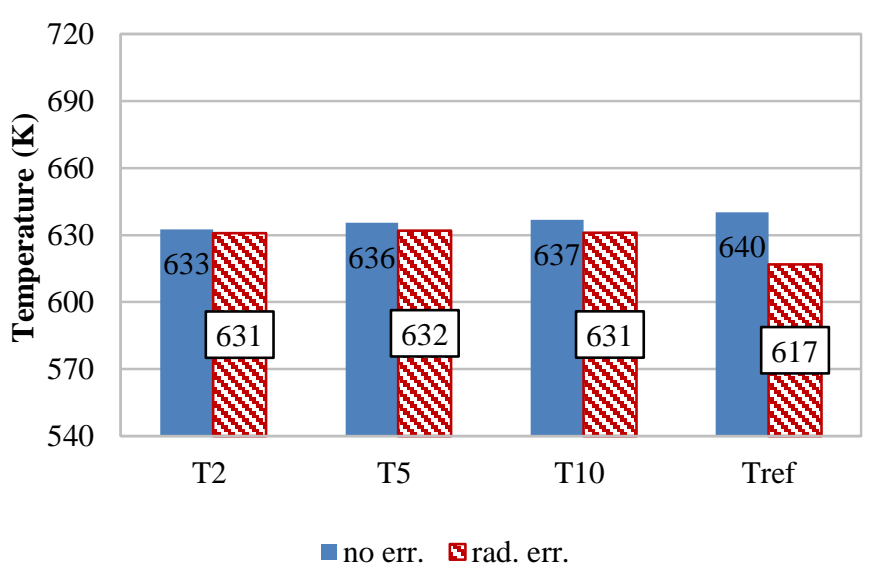

Figure 10: Effect of radiation error (rad err.) on time-averaged temperatures for all thermocouple sizes at the $1500 \mathrm{rpm} / 6.8 \mathrm{bar}$ nIMEP condition. Constant wall temperature, $T_{w}=509 \mathrm{~K}$.

The results clearly indicate that radiation has a small effect on the fine-wire thermocouples for the conditions modelled here. Due to its relatively larger size the error due to radiative heat transfer on the reference thermocouple is $\sim 23 \mathrm{~K}$. The effects of thermal inertia discussed in the previous section are still apparent for the 3-TC probe thermocouples, with the T5 thermocouple indicating the highest temperature, but it is interesting to note though that the 3-TC thermocouples are now indicating higher time-average temperatures than the reference thermocouple. Due both to the relatively minor effect of radiation on the time-averaged temperature and to the fact that the results in Figure 10 now follow an opposite trend to those shown in Figure 8, i.e. the reference thermocouple reports a lower time-average temperature than the 3-TC probe values, we conclude that the substantial differences in the experimental time-averaged temperatures reported by the 3-TC and reference thermocouples are not due to the effects of radiative heat transfer.

\section{Radiation and conduction terms included}

Equation (6) shows the discretised solution of Equation (2) for $T_{S}$ with both radiation and conduction terms included

$$
\begin{aligned}
T_{s}(t+1)=T_{s}(t)+ & \frac{\Delta t}{m c_{p}}\left[h A_{S}\left(T_{g}(t)-T_{s}(t)\right)\right. \\
& -\sigma \varepsilon A_{S}\left(T_{s}^{4}(t)-T_{w}^{4}\right)-\frac{2 k A_{c s a}}{L}\left(T_{s}(t)\right. \\
& \left.\left.-T_{w}\right)\right]
\end{aligned}
$$

The estimation of steady state conduction error is commonly carried out by treating the thermocouple as a thin rod and applying the analytical solutions available for fins [32, 35]. However, the effect of conduction error on unsteady temperature measurements is not well covered in the literature. In a study on unsteady temperature at the wake of a heated cylinder, Khine et al. [25] corrected for the conduction error along the body of the sensor by assuming a linear temperature variation along the length of the probe. The authors take a similar approach in their discretisation, although here the conduction term is multiplied by a factor of 2 to account for the two wires of the thermocouple junction. Note that the use of a single value for thermal conductivity $k$ is not expected to lead to significant error where K-type thermocouples are concerned as the thermal conductivities of Alumel and Chromel are quite similar (29.7 and 17 $\mathrm{W} / \mathrm{mK}$ respectively [36]).

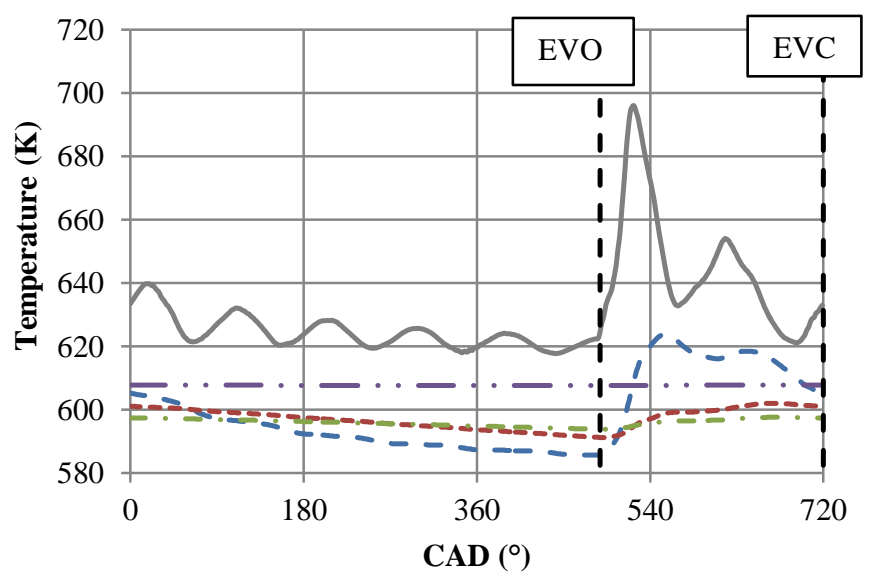

$$
---\mathrm{T} 2 \ldots--\mathrm{T} 5-\cdots \mathrm{T} 10-\cdots \mathrm{Tref} \longrightarrow \mathrm{Tg}
$$

Figure 11: Thermocouple modelling results including conduction and radiation losses for the $1500 \mathrm{rpm} / 6.8 \mathrm{bar}$ nIMEP conditions (Equation 6). The wall temperature was constant at $T_{w}=509 \mathrm{~K}$.

The results of Equation 6 for the $1500 \mathrm{rpm} / 6.8$ bar nIMEP case can be seen in Figure 11. Comparing against the results presented in Figure 9 it is clear that heat transfer along the sensor's body has a significant impact on the resulting temperature measurement for all thermocouples, with the conduction error having a more profound effect on the fine-wire thermocouples than the reference sensor. More specifically, the results presented in Figure 11 indicate similar trends to the experimental results presented in Figures 7 and 8; namely the time-average temperature of the fine-wire thermocouples is lower than the reference thermocouple.

The relative magnitudes of the conduction effects on the fine-wire and reference thermocouples observed in these simulations is in sharp contrast to the literature where conduction effects in unsteady flows with fine-wire thermocouples is usually ignored due to the small diameter to length ratio of these sensors [13], [19]. On first sight, according to Equation (6) one would expect the opposite trend, as the conduction error is directly proportional to the cross-sectional area of the thermocouple wires (assuming constant thermal properties) [25, 37]. However, each term in Equation 6 is also dependent on the thermal mass $\left(m c_{p}\right)$ of the thermocouple junction. Looking into the relative contribution of each heat transfer term in Equation (6) to the total energy of the thermocouple junction can assist in understanding the observed thermocouple behaviour. Figure 12 shows this relative contribution in percentage terms for the reference thermocouple. Following Equation (6), a positive sign for the radiation and conduction terms will indicate the energy transferred from the control volume (Figure 5) whereas a positive sign for the convective term shows the energy flow into the control volume. 


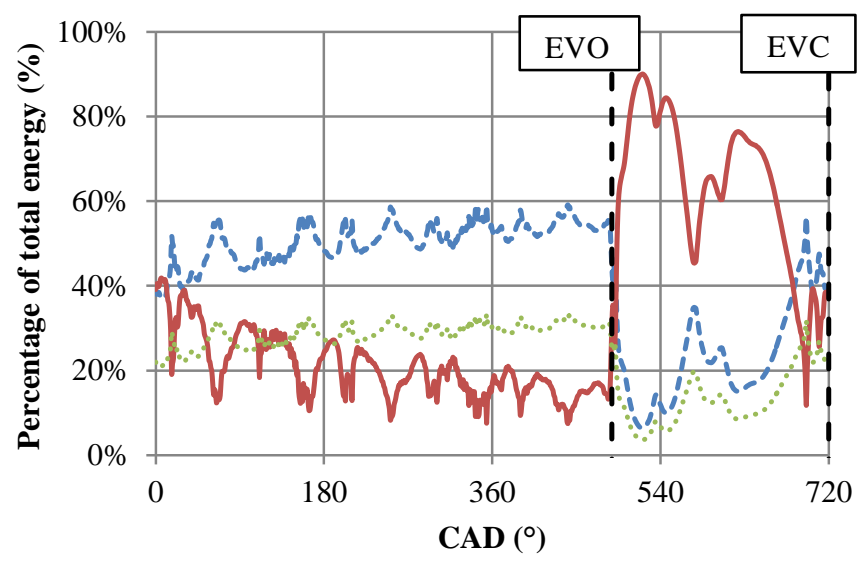

-----Qrad. — Qcnv. ........ Qcnd.

Figure 12: Relative contributions of heat transfer modes on the total energy of the reference thermocouple junction during one cycle.

As expected, the dominant heat transfer mode during the time that the exhaust valves are open is heat convection from the hot gases to the thermocouple surface. During this period heat conduction and radiation have a very small contribution in the total energy transferred. However, once the valves are closed, the conductive and radiative terms are significantly increased, with the convective term progressively decreasing to $\sim 15 \%$ - this is in agreement with observations made by Cambel and Jennings [38] where they concluded that as the flow velocity is reduced the temperature measurement error is dominated by radiative and conductive heat transfer. Under these conditions, the effect of radiation is much more pronounced compared to the heat conducted along the length of the probe, with the maximum values approaching $\sim 55 \%$ and $\sim 30 \%$ of the instantaneous energy transfer for the radiation and conduction terms respectively.

The results for the relative contribution of heat transfer modes for T2 thermocouple are presented in Figure 13. An interesting observation here is the opposite trend that the conduction and convection terms follow during the EVC period for the two thermocouples conduction is increasing during the EVC event for the reference thermocouple and decreasing for the $\mathrm{T} 2$ thermocouple and vice-versa for the convection term. The radiation term is constant irrespective of junction size for this period. During the EVC event the temperature of the gas will keep decreasing as it exchanges heat with its cooler surrounding, a phenomenon that is observed experimentally in Figure 7. With respect to the sensor operation and the reported gas temperature three scenarios now exist:

1. the gas and sensor cool at the same rate

2. the gas cools faster than the sensor, in which case that gas and sensor temperatures will converge - which will tend to reduce the magnitude of the convection term and relatively increase the magnitude of the conduction and radiation term as is seen in Figure 12 to be the case for the reference thermocouple

3. the sensor cools faster than the gas - increasing the magnitude of the convection term and reducing the relative magnitude of the conduction term as is shown to be the case for the T2 thermocouple in Figure 13.

Referring back to Figure 11, it is clear that the temperature of the T2 thermocouple is reducing at a faster rate than the gas temperature during the EVC period. This indicates that the T2 thermocouple loses heat more readily (despite the conduction term being of similar magnitude to the reference thermocouple during EVC) due to its lower thermal mass. The compounded effects of lower thermal inertia and conduction losses then explain the lower time-average temperature of the fine-wire thermocouples compared to the reference thermocouple that has been observed experimentally (Figures 7 and 8).

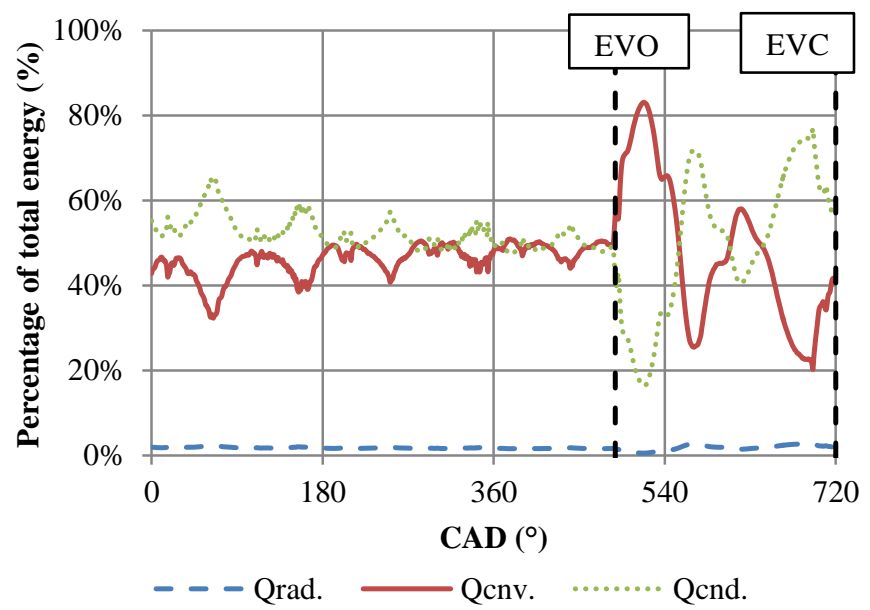

Figure 13: Relative contributions of heat transfer modes on the total energy of the $\mathrm{T} 2$ thermocouple junction during one cycle.

The modelling work presented above has highlighted the significant effects of conduction, and the relatively minor effect of radiation when fine-wire thermocouples are used (the effect of radiation is more pronounced for the $3 \mathrm{~mm}$ thermocouple - see Figure 12) in the measurement of exhaust gas temperature on internal combustion engines. The results have shown that time-averaged exhaust gas temperatures are significantly under-estimated by uncorrected finewire thermocouple measurements. Nevertheless, it is recognised that the faster transient response of the fine-wire devices does offer the potential of recovering the instantaneous exhaust gas temperature and hence, if required, the correct time-averaged temperature. The following section presents the initial results of an ongoing study on dual probe temperature reconstruction methods for engine applications

\section{Dynamic error compensation on unsteady temperature measurements}

The numerical work presented above indicates clearly that even the smallest thermocouple will introduce some dynamic error due to its thermal inertia. Temperature reconstruction thus involves the process of recovering the high-frequency temperature components that have been lost due to the thermal inertia of the thermocouple, by estimating the thermocouple's time-constant.

\section{Numerical method}

Dual probe techniques assume that the two thermocouples are exposed to the same temperature and velocity fields, thus the gas temperature at the thermocouples' location is assumed to be the same. The time-constants can then be estimated by solving a simultaneous system of equations for each thermocouple based on an energy balance approach. The compensation method used here is based on the work of Tagawa and co-workers [14], who argued that 
the local gas temperature for the two thermocouples will rarely be the same and thus suggested to minimise the time-average difference between the two values using a least squares minimisation criterion. Treating the thermocouples as a first order lag system and ignoring conduction and radiation we have the following system of equations:

$$
\left.\begin{array}{l}
T_{g 1}=T_{s 1}+\tau_{1} \frac{d T_{s 1}}{d t}, \\
T_{g 2}=T_{s 2}+\tau_{2} \frac{d T_{s 2}}{d t},
\end{array}\right\}
$$

Minimizing the time-averaged temperature difference between the two local gas temperatures (i.e. $T_{g 1}$ and $T_{g 2}$ ) results in the timeconstants $\tau_{1}$ and $\tau_{2}$ presented in Equation 8 .

$$
\left.\begin{array}{rl}
\tau_{1} & =\frac{\left(\sum G_{2}^{2}\right)\left(\sum G_{1} \Delta T\right)-\left(\sum G_{2} \Delta T\right)\left(\sum G_{1} G_{2}\right)}{\left(\sum G_{2}^{2}\right)\left(\sum G_{1}^{2}\right)-\left(\sum G_{1} G_{2}\right)^{2}}, \\
\tau_{2} & =\frac{\left(\sum G_{1} G_{2}\right)\left(\sum G_{1} \Delta T\right)-\left(\sum G_{2} \Delta T\right)\left(\sum G_{1}^{2}\right)}{\left(\sum G_{2}^{2}\right)\left(\sum G_{1}^{2}\right)-\left(\sum G_{1} G_{2}\right)^{2}},
\end{array}\right\}
$$

Where $\Sigma$ indicates a moving averaging processes and $\mathrm{G}$ the rate of change of the thermocouple signal (i.e. $\frac{d T_{s}}{d t}$ ).

The results of Equation (8) are highly sensitive to the detail of the averaging process. Tagawa et al., [14] employed a central sliding moving average window, with the choice of window size providing a compromise between temporal resolution and non-physical values from the resulting time-constants (i.e. negative values for $\tau$ ). A small window size will highlight the temporal characteristics of the timeconstants however, in some instances it can only include high frequency temperature components and consequently the minimisation criterion will try to compensate for these temperature fluctuations by providing a negative time constant. On the other hand, as the window size is increased, the effect of averaging is more pronounced and the resulting time-constants approach their time average $(\bar{\tau})$ rather their instantaneous values $(\tau)$.

In this work, we note that the gas dynamics in a single cylinder engine exhaust manifold are significantly different in the EVC period and the EVO period. Accordingly, these time periods are considered separately in order to reduce the effect of averaging on the instantaneous values of $\tau$, especially during the blowdown period that is of highest interest. Furthermore, for the EVO period, the timeconstants were estimated at each time-step based on past values, using an exponential moving average window following Equation (9) [39].

$$
f(t)=\alpha g(t)+(1-\alpha) f(t-1)
$$

Where $f(\mathrm{t})$ is the filtered output, $g(\mathrm{t})$ is the input value and $\alpha$ is the weight factor. This weight factor determines the importance of the past values on the filtered output, with a value of 1 implying that the same weight is given to all past data (i.e. simple moving average). Exponential moving average methods are generally preferred over a simple moving average in predictive models to better capture rapidly changing values. In the period that the exhaust valve is open, and particularly close to the blowdown period, the time-constant will be rapidly changing and thus the exponential moving average method is appropriate. The value of $\alpha$ for the exponential moving averaging was set to $\alpha=0.08$ for the EVO event.

Page 9 of 12
During the time-averaging period, the time-constants are assumed to be kept constant with the window size during the EVC period set to half the number of points during this time. This is due to the fact that the gas dynamics during this time are not expected to be significant (i.e. no flow condition) and hence an average time-constant for this period would be acceptable.

\section{Reconstruction Results}

The accuracy of the reconstruction method and the effects of the averaging methods have been assessed by taking the thermocouple signals, comprising the fine-wire thermocouple probe (i.e. T2, T5 and T10 thermocouples), modelled by Equation (4) and attempting to reconstruct the gas temperature provided by the 1-D model. Prior results have shown that the reconstruction method requires the dynamic response of the thermocouple pair that is used to be sufficiently different so that at least one thermocouple can capture the necessary flow information. A diameter ratio of more than 2.5 between the thermocouple pair has been recommended [14]. The results presented here are calculated using the pair of T2/T5 thermocouples, with the T2/T10 pair comparatively showing a good agreement.

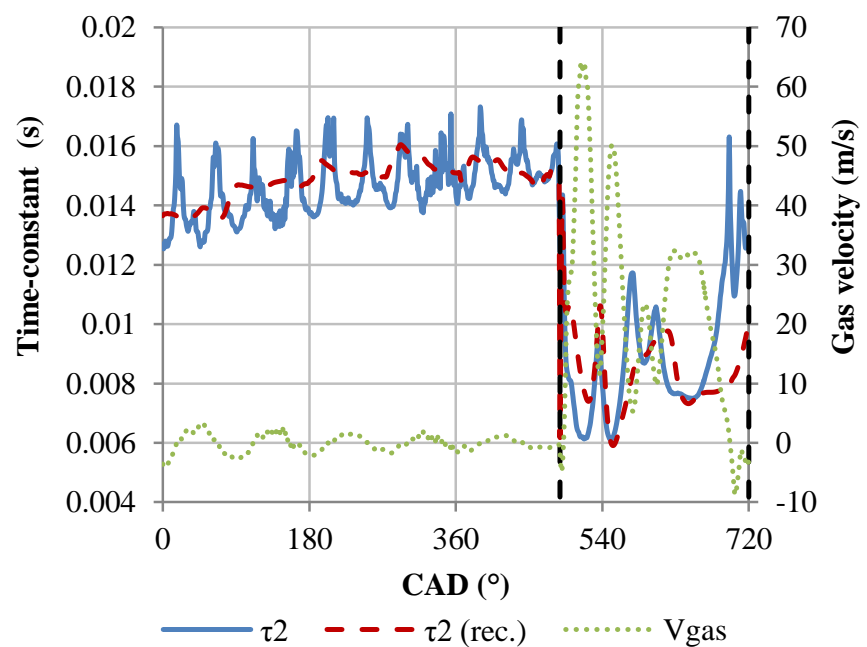

Figure 14: Reconstructed time-constant for the T2 thermocouple for the $1500 \mathrm{rpm} / 6.8 \mathrm{bar}$ nIMEP case. The solid blue line indicates the true time-constant values as calculated from the lumped capacitance model.

Figure 14 shows the calculated time-constant $\left(\tau_{\text {rec }}\right)$ for the T2 thermocouple according to Equation (8). The solid line represents the "true" time-constant as calculated from the lumped capacitance model following Equation (1) and the dotted line indicates the 1-D exhaust gas velocity. For the EVC period the reconstructed timeconstant follows the true value well, however without capturing the peaks and troughs due to averaging. At the time of the EVO, a sudden drop in the time-constant is observed, which is in agreement with the fast moving gases and the high heat transfer coefficient values during blowdown, reaching a minimum value of $\sim 6 \mathrm{~ms}$. At around $580^{\circ} \mathrm{CA}$ the time-constant reaches a local maximum before starting to reduce again, indicating the second part of the EVO event, where the gases are being pushed out of the cylinder due to the piston movement. The erratic behaviour observed at the end of the cycle prior to EVC is due to gas flow-reversal and it is an artefact of the 1D model, as can be seen from the profile of the exhaust gas velocity. In general, the reconstructed time-constant follows the rapid changes during the EVO period, with a small discrepancy observed during the 
point of expected maximum temperature (i.e. $528^{\circ} \mathrm{CA}$ ). The reconstructed time-constant for the T5 thermocouple is a similarly good match to the "true" value.

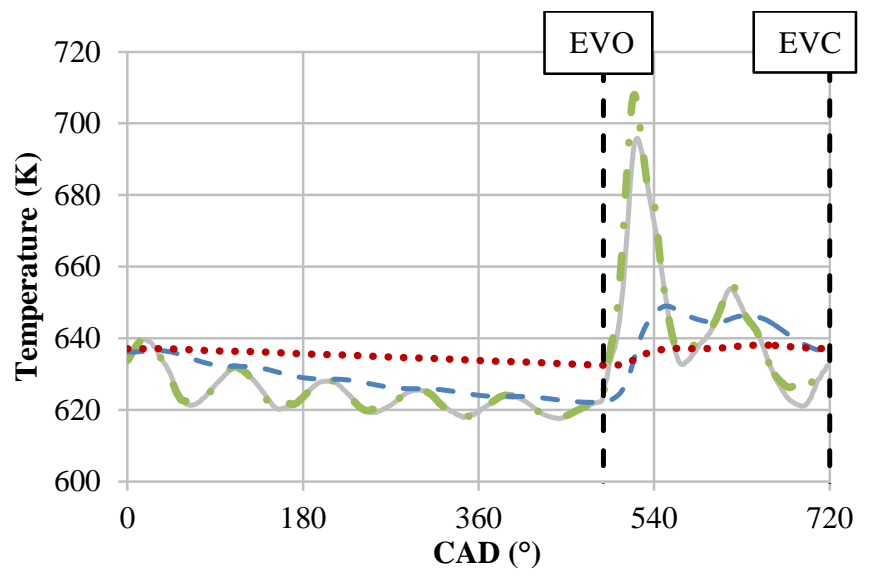

T Tgas $-\cdots \mathrm{T}($ rec.) $\quad$ - $-\mathrm{T} 2 \quad \ldots$ T5

Figure 15: Reconstructed gas temperature for the $1500 \mathrm{rpm} / 6.8$ bar nIMEP case. The solid line indicates the gas temperature from the 1-D model.

Figure 15 shows the results of the resultant temperature reconstruction for the $1500 \mathrm{rpm} / 6.8$ bar nIMEP case. The dashed and dotted lines represent the $\mathrm{T} 2$ and the $\mathrm{T} 5$ thermocouples respectively. During the EVC event the reconstructed temperature is line-on-line with the 1-D model results, which indicates that the choice of a large window size due to the slow gas dynamics for this period is justified. Good agreement is also observed during the EVO period, albeit with a $\sim 1.5 \%$ over-prediction in peak temperature. This is attributed to the larger time-constant estimated at this point (Figure 14) which consequently leads to bigger dynamic error compensation according to Equation (6). Note however that this error in peak temperature is within the error limits of the sensor under these conditions. Similar results were also observed for the $1750 \mathrm{rpm} / 13.5$ bar nIMEP case however, the discrepancy in peak temperature was $\sim 2 \%$. These results are significant. When the reconstructed values were applied to a recently published energy balance model [7] the resultant timeaveraged exhaust enthalpy estimation was changed by 5 percentage points (i.e. from $30 \%$ to $35 \%$ of the total input energy) compared to the case where the uncorrected time-averaged temperature is used.

\section{Future work}

The reconstruction results presented above show great promise but as yet do not include compensation for radiation or conduction losses. Having highlighted the importance of conduction in this present study, the authors are currently extending the reconstruction method to include a conduction correction. The results of this work will be presented in future publications.

\section{Summary/Conclusions}

Detailed experimental and modelling work has been carried out to identify the response of different thermocouple sizes on the temperature measurement of unsteady flows, along with their resulting measurement errors. As such, a standard $3 \mathrm{~mm}$ sheathed thermocouple, used as a reference sensor, was installed on the exhaust manifold of an ICE along with three fine-wire thermocouples of varying diameter and tested under two different speed/load conditions.

The experimental results have shown that the fine-wire thermocouples indicate a lower time-average temperature compared to the sheathed thermocouple under both speed/load conditions, with the maximum discrepancies ranging from $\sim 40-80 \mathrm{~K}$ depending on test conditions. A thermocouple model was built in order to understand the causes of this behaviour. The model results show that:

1. Radiation error is not important for EGT when using finewire thermocouples based on the conditions tested here

2. Conduction error has a significant effect on the measurement of unsteady temperatures

3. The effect of conduction error has a more pronounced effect on the fine-wire thermocouples due to their lower thermal mass as they can lose heat more readily.

4. When fine-wire thermocouples are used for time-average temperature measurements of unsteady flows they will indicate a lower temperature compared to a sheathed thermocouple due to the compounded effects of conduction error and lower thermal mass.

A temperature reconstruction technique has also been presented to correct for the dynamic error induced by the thermocouples without taking into account the radiation and conduction errors. The results of the reconstruction show a very good agreement with the "true" gas temperature with a maximum error on peak temperature of $\sim 1.5-2 \%$ depending on speed/load condition, which improves the results of the time-average exhaust enthalpy estimation by $17 \%$. As the importance of the conduction error has been highlighted, future work will extend the reconstruction method to include the effects of conduction error on the temperature reconstruction.

\section{References}

1. Heuer, T., et al., Numerical and Experimental Analysis of the Thermo-Mechanical Load on Turbine Wheels of Turbochargers. 2006(42401): p. 325-332.

2. Mollenhauer, K., Measurement of Instantaneous Gas Temperatures for Determination of the Exhaust Gas Energy of a Supercharged Diesel Engine. 1967, SAE International.

3. Tsinoglou, D.N. and G.C. Koltsakis, Potential of thermal methods for catalyst on-board diagnosis. Proceedings of the Institution of Mechanical Engineers, Part D: Journal of Automobile Engineering, 2002. 216(7): p. 565-579.

4. Fleming, W.J., Overview of automotive sensors. IEEE Sensors Journal, 2001. 1(4): p. 296-308.

5. Stone, R., Introduction to Internal Combustion Engines. 4th ed. 2012, UK: Palgrave Macmillan.

6. Mavropoulos, G.C., Unsteady Heat Conduction Phenomena in Internal Combustion Engine Chamber and Exhaust Manifold Surfaces. Heat Transfer - Engineering Applications, ed. V. Vikhrenko. 2011, Croatia: Intech.

7. Papaioannou, N., et al., Evaluation of exhaust gas recirculation techniques on a high-speed direct injection diesel engine using first law analysis. Proceedings of the Institution of Mechanical Engineers, Part D: Journal of Automobile Engineering. 0(0): p. 0954407017749110. 
8. Dahlstrom, J., et al., Experimental Comparison of Heat Losses in Stepped-Bowl and Re-Entrant Combustion Chambers in a Light Duty Diesel Engine. 2016, SAE International.

9. Olmeda, P., et al., Evaluation of EGR Effect on the Global Energy Balance of a High Speed DI Diesel Engine. 2016, SAE International.

10. Smith, L.A., et al., Application of a First Law Heat Balance Method to a Turbocharged Automotive Diesel Engine. 2009, SAE International.

11. Caton, J.A., Comparisons of Thermocouple, TimeAveraged and Mass-Averaged Exhaust Gas Temperatures for a Spark-Ignited Engine. 1982, SAE International.

12. Omega, 2018 [online], https://www.omega.co.uk/ (Accessed 2 March 2018).

13. Kar, K., et al., Instantaneous Exhaust Temperature Measurements Using Thermocouple Compensation Techniques. 2004, SAE International.

14. Tagawa, M. and Y. Ohta, Two-thermocouple probe for fluctuating temperature measurement in combustionRational estimation of mean and fluctuating time constants. Combustion and Flame, 1997. 109(4): p. 549-560.

15. Dupont, A., et al., Influence of temperature on the frequency response of fine-wire thermocouples over the range $(300 \mathrm{~K}-800 \mathrm{~K})$ in airflows. Journal of Physics E: Scientific Instruments, 1984. 17(9): p. 808.

16. Heitor, M.V., A.M.K.P. Taylor, and J.H. Whitelaw, Simultaneous velocity and temperature measurements in a premixed flame. Experiments in Fluids, 1985. 3(6): p. 323339.

17. Hopkins, K.C., J.C. Larue, and G.S. Samuelsen, Effect of Mean and Variable Time Constant on Compensated Thermocouple Measurements, in Instrumentation for Combustion and Flow in Engines, D.F.G. Durão, J.H. Whitelaw, and P.O. Witze, Editors. 1989, Springer Netherlands: Dordrecht. p. 55-68.

18. Forney, L.J. and G.C. Fralick, Three-wire thermocouple: Frequency response in constant flow. Review of Scientific Instruments, 1995. 66(5): p. 3331-3336.

19. Kee, R.J., et al., Measurement of Exhaust Gas Temperatures in a High Performance Two-Stroke Engine. 1998, SAE International.

20. Kar, K., A.K. Swain, and R. Raine, Identification of TimeVarying Time Constants of Thermocouple Sensors and Its Application to Temperature Measurement. Journal of Dynamic Systems, Measurement, and Control, 2008. 131(1): p. 011005-011005-10.

21. West, W.E. and J.W. Westwater, Radiation-Conduction Correction for Temperature Measurements in Hot Gases. Industrial \& Engineering Chemistry, 1953. 45(10): p. 21522156.

22. Bradley, D. and K.J. Matthews, Measurement of High Gas Temperatures with Fine Wire Thermocouples. Journal of Mechanical Engineering Science, 1968. 10(4): p. 299-305.

23. Shaddix, C.R., Correcting thermocouple measurements for radiation loss: A critical review. Conference: 33rd National Heat Transfer Conference NHTC'99, Albuquerque, NM (US), 08/15/1999--08/17/1999; 1999,New York, NY (US); Sandia National Labs, Paper NHTC99.282.

24. Kobus, C.J., True fluid temperature reconstruction compensating for conduction error in the temperature measurement of steady fluid flows. Review of Scientific Instruments, 2006. 77(3): p. 034903.

25. Khine, S.M., T. Houra, and M. Tagawa, Heat-conduction error of temperature sensors in a fluid flow with nonuniform and unsteady temperature distribution. Review of Scientific Instruments, 2013. 84(4): p. 044902.

26. Leach, F., et al., The effect of a stepped lip piston design on performance and emissions from a high-speed diesel engine. Applied Energy, 2018. 215: p. 679-689.

27. Burns, G.W., et al., The Calibration of Thermocouples and Thermocouple Materials. 1989: U.S. Department of Commerce, National Institute of Standards and Technology.

28. NIST. NIST ITS-90 Thermocouple Database.2000 [Online], https://srdata.nist.gov/its90/main/its90_main_page.html (Accessed 2 March 2018).

29. Demuynck, J., A Fuel Independent Heat Transfer Correlation for Premixed Spark Ignition Engines, in Faculty of Engineering and Architecture. 2012, Ghent University: Ghent, Belgium.

30. Whitaker, S., Forced convection heat transfer correlations for flow in pipes, past flat plates, single cylinders, single spheres, and for flow in packed beds and tube bundles. AIChE Journal, 1972. 18(2): p. 361-371.

31. Ran Fu, T., J. Bin Tian, and H. Sheng Wang, Apparent Emissivity of Combustion Soot Aggregate Coating at High Temperature. Journal of Heat Transfer, 2017. 139(4): p. 042701-042701-7.

32. Eckert, E.R.G., Drake R.M., Heat and Mass Transfer. 1974, New Delhi: TATA McGraw-Hill.

33. Gamma Technologies, 2018 [online] https://www.gtisoft.com/ (Accessed 2 March 2018)

34. Leach FCP, Davy MH, Siskin D, Pechstedt R, and Richardson D, "An optical method for measuring exhaust gas pressure from an internal combustion engine at high speed". Review of Scientific Instruments. Vol 88, Issue 12 125004. doi:10.1063/1.5005161

35. Sparrow, E.M., Measurements in heat transfer: Error estimates in temperatue measurements. $2 \mathrm{~d}$ ed. ed. Series in thermal and fluid engineering, ed. E.R.G.E.a.R.J. Goldstein. 1976, Washington Hemisphere Pub. Corp.

36. AZO Materials, [Online], https://www.azom.com/ (Accessed 2 March 2018).

37. Warren, R.C., Design of Thermocouple Probes for Measurement of Rocket Exhaust Plume Temperatures. 1994, Aeronautical and Maritime Research Laboratory: Melbourne, Australia.

38. Campbell, A.B.a.J., B.H., Gas Dynamics. 1958, McGrawHill: New York.

39. Ross, S.M., Introduction to probability and statistics for engineers and scientists. 1987, New York: John Wiley and Sons.

\section{Contact Information}

Nick Papaioannou

Department of Engineering Science, University of Oxford, Parks Rd, Oxford, OX1 3PJ, UK

Email: nick.papaioannou@eng.ox.ac.uk

\section{Acknowledgments}

The authors would like to thank the Jaguar Land Rover Limited and University of Oxford John Fell fund for financial support. Nick Papaioannou would like to thank EPSRC for supporting his research (Grant number: 1515450). The authors would also like to thank BP 
International for supplying the Diesel. Leo Fang, Gilbert Sammut, Adam Weall and Dave Richardson are acknowledged for technical support. Finally, the authors would like to thank Liyah Dholiwar for undertaking much of the engine testing and the Dept. of Engineering Science technicians and maintenance teams for facilities support.

\section{Definitions/Abbreviations}

CAD

CFD

DAQ

DPF

EGT

EVC

EVO

ICE
Crank Angle Degrees

Computational Fluid

Dynamics

Data Acquisition

Diesel Particulate Filter

Exhaust Gas Temperature

Exhaust Valve Closing

Exhaust Valve Opening

Internal Combustion Engine
Pumping Mean Effective

\section{Pressure}

SCR
Selective Catalytic

Reduction 